\title{
La búsqueda de atención para la tuberculosis en Chiapas, México
}

\author{
Guadalupe del Carmen Álvarez Gordillo, ${ }^{1}$ \\ José Eugenio Dorantes Jiménez ${ }^{2}$ y Dolores Molina Rosales ${ }^{1}$
}

RESUMEN Objetivos. Analizar el proceso de búsqueda de atención a la tuberculosis y su influencia en la adherencia al tratamiento en Chiapas, dado que el diagnóstico temprano y la adherencia al tratamiento son los factores principales para el éxito de los programas de control de la tuberculosis.

Métodos. Se realizó un estudio cualitativo mediante entrevistas grupales a 11 grupos de pacientes de las regiones Centro, Altos y Fronteriza del estado de Chiapas, México.

Resultados. Se detectaron diferentes alternativas a las que los enfermos acuden para su atención. Los enfermos manifestaron un retraso importante en el diagnóstico por problemas del mismo enfermo y de la atención recibida en los servicios de salud. Las opciones de tratamiento que siguieron fueron el resultado de las percepciones que tienen sobre las causas de la enfermedad y de la diversidad de etnomedicinas legitimadas en las comunidades.

Conclusiones. El poco conocimiento sobre la enfermedad propicia la elección de diferentes alternativas para su atención. El control de la tuberculosis requiere una utilización óptima de los servicios de salud en Chiapas y un programa de educación en materia de salud, tomando en cuenta la realidad social, cultural y económica en la que vive la población.

Palabras clave Tuberculosis, diagnóstico, tratamiento, adherencia al tratamiento, búsqueda de atención.

La tuberculosis ha resurgido como problema de salud mundial debido a la aparición de fuentes infectantes con cepas bacterianas resistentes, atribuible al uso incorrecto de los antibióticos, al fracaso del tratamiento no supervisado y al manejo inapropiado de los programas de control. Además, se ha agregado la infección por el virus

1 El Colegio de la Frontera Sur (ECOSUR). Toda la correspondencia debe enviarse a Guadalupe del Carmen Álvarez Gordillo, Carretera Panamericana y Periférico Sur s/n. CP 29290 San Cristóbal de las Casas, Chiapas, México. Correo electrónico: galvarez@sclc.ecosur.mx

2 Instituto de Salud del Estado de Chiapas. Tuxtla Gutiérrez, Chiapas, México. de la inmunodeficiencia humana (VIH) como un desencadenante poderoso de la epidemia de tuberculosis (1). Lo anterior, aunado a la pobreza y a las desigualdades crecientes entre ricos y pobres, sobre todo en países en vías de desarrollo, hace que tanto los tratamientos individuales como las campañas de control sean cada vez más difíciles y de alto costo humano y social (2-4). La eficacia ${ }^{3}$ del tratamiento farmacológico primario frente

\footnotetext{
3 La eficacia es el porcentaje de pacientes que se consideran curados al término de un tratamiento completo.
}

a cepas de Mycobacterium tuberculosis sensibles a los medicamentos es del $98 \%(5,6)$, pero disminuye sustancialmente cuando los medicamentos se toman inapropiadamente o se abandona el tratamiento.

En México, de 1993 a 1998 se aprecia un incremento de $24 \%$ en el número de casos de tuberculosis pulmonar, y de 33,5\% en las tasas de morbilidad, que pasaron de 14 a 18,7 casos por cada 100000 habitantes (7). La mortalidad por tuberculosis fue de 3,27 defunciones por 100000 habitantes. En Chiapas, en 1998, se registraron tasas de incidencia y mortalidad del 41,7 y 8,2 por 100000 habi- 
tantes, respectivamente, ocupando el primer lugar en mortalidad por tuberculosis de todos los estados de la República de México, con tasas superiores al doble de las nacionales. Durante el período de 1989 a 1996, más de $95 \%$ de los nuevos casos de tuberculosis pulmonar diagnosticados iniciaron tratamiento, y solamente 73,2\% lo terminó. Se registraron abandonos del tratamiento en $18 \%$ de los pacientes y fracasos en $8 \%$. La eficacia alcanzó solamente $90,3 \%$, lo que podría implicar la falta de supervisión del tratamiento y la presencia de cepas de $M$. tuberculosis resistentes a los medicamentos primarios. La eficiencia ${ }^{4}$ del tratamiento en las regiones Centro, Altos y Fronteriza fue de $68,6,72,1$ y $69,7 \%$, respectivamente (8).

La población de las tres regiones estudiadas en Chiapas es de 1663473 habitantes, con una densidad poblacional de 70,6 habitantes por $\mathrm{km}^{2}$. El $49,5 \%$ de la población está ocupada en el sector primario de la economía, $19,2 \%$ no tiene ingresos y $37,4 \%$ recibe menos de un salario mínimo. El analfabetismo es de 20, 38 y 26\% en las regiones Centro, Altos y Fronteriza, y el porcentaje de hablantes de alguna lengua indígena es de 6,2, 57,6 y 13,8\%, respectivamente (9).

En relación con los servicios de salud, la cobertura de atención es de 93,5\%. El 19,6\% de la población está asegurada en instituciones como el Instituto Mexicano del Seguro Social (IMSS), el Instituto de Servicios y Seguridad Social para los Trabajadores del Estado (ISSSTE) o el Instituto de Seguridad Social para los Trabajadores del Estado de Chiapas. El 72,7\%, considerada población sin derecho formal a servicios médicos, recibe atención institucional de las unidades de la Secretaría de Salud y de IMSSSolidaridad, y 1,4\% recibe atención médica privada (10).

Los servicios del Programa de Prevención y Control de la Tuberculosis

\footnotetext{
4 La eficiencia es el porcentaje de pacientes considerados como curados del total que inician un tratamiento.
}

(detección, diagnóstico, tratamiento) son gratuitos y están disponibles en todas las unidades de salud y mediante promotores de salud. La detección de casos es pasiva, es decir, entre los enfermos que acuden con sintomatología respiratoria a los establecimientos de salud.

En investigaciones realizadas en el estado de Chiapas se ha documentado que el Programa de Control de la Tuberculosis en las zonas indígenas no ha logrado una implantación óptima, debido a que las prácticas culturales de la población limitan la aceptación de los servicios médicos $(11,12)$. Otros estudios señalan la falta de medicamentos, además de la insuficiente supervisión del personal paramédico en los programas y el abandono del tratamiento (13). ${ }^{5}$

Se eligió la información generada por 11 grupos de discusión, ya que esta técnica permite obtener un discurso global de algún evento o proceso social, en este caso de la tuberculosis, su detección, búsqueda de atención y tratamiento. Nos interesaba conocer lo que los pacientes que acudieron a diferentes instituciones de salud para la atención de la tuberculosis podían contar de sus experiencias, e identificar los puntos en común, pese a lo diversas que pudieran ser sus vivencias.

Entre los modelos del proceso salud-enfermedad se encuentra la perspectiva de la antropología médica, que plantea una propuesta construccionista según la cual todo padecimiento constituye un proceso social e histórico que necesita ser reconstruido para poder comprender sus significados actuales, no solo para la población, sino también para el equipo de salud (14). Así, "según sean las condiciones de vida y de trabajo de los individuos en ciertos momentos históricos, es decir, según sea la clase y el grupo social específico al que pertenezca en una determinada formación social, será el tipo de enfermedades

\footnotetext{
5 Tuberculosis and Health Care in Highland Chiapas, México: An ethnographic study. Documento mimeografíado. Centro de Investigaciones en Salud de Comitán, 1991.
}

que se padezcan, su gravedad y su frecuencia, así como el acceso real a los servicios médicos, la esperanza de vida y de qué mueren los individuos" $(15,16)$.

El objetivo de este estudio fue profundizar por métodos cualitativos en el proceso de búsqueda de atención de la tuberculosis en Chiapas y dar pautas para generar estrategias de prevención y control acordes con las necesidades de los enfermos y los servicios de salud disponibles.

\section{MATERIALES Y MÉTODOS}

\section{Diseño del estudio}

Se llevó a cabo un estudio cualitativo mediante entrevistas grupales a pacientes con tuberculosis de las regiones Centro, Altos y Fronteriza del estado de Chiapas. Se formaron 11 grupos de pacientes con tuberculosis diagnosticada por baciloscopia durante el último trimestre de 1997 y el primer trimestre de 1998 (cuadro 1). El trabajo de campo se dividió en dos fases. Se hizo una primera ronda de entrevistas en las que se aplicó la guía previamente diseñada para el estudio (anexo 1). Posteriormente se realizó una segunda serie de entrevistas para profundizar en algunos temas y discutir con los propios informantes lo dicho en la primera fase.

Todas las entrevistas se realizaron en lugares independientes de los centros de salud, salvo las dos primeras reuniones con los informantes de la región Fronteriza. ${ }^{6}$ Las sesiones con cada grupo tuvieron una duración de 2 horas. Las entrevistas se iniciaron con la presentación de los participantes y enseguida se hicieron preguntas abiertas, dejando que las respuestas se extendieran según sus experiencias. Con autorización de los pacientes, las entrevistas fueron grabadas por un equipo especializado y se transcribieron por completo.

\footnotetext{
6 Esas dos reuniones se hicieron en el Centro de Salud de Comalapa, puesto que fue el único lugar de referencia ubicado por los informantes y los investigadores.
} 
CUADRO 1. Características generales de los participantes en las entrevistas grupales. Chiapas, México, 1998

\begin{tabular}{|c|c|c|c|c|c|}
\hline Grupo & $\begin{array}{l}\text { Número de } \\
\text { integrantes }\end{array}$ & Municipio de residencia & $\begin{array}{l}\text { Edad promedio } \\
\text { (años) }\end{array}$ & $\begin{array}{l}\text { Pacientes con } \\
\text { abandonos }\end{array}$ & Ocupación \\
\hline 1. Comalapa & 9 hombres & $\begin{array}{l}\text { Comalapa } \\
\text { Bellavista }\end{array}$ & 31,3 & 1 & $\begin{array}{l}8 \text { agricultores } \\
1 \text { comerciante }\end{array}$ \\
\hline 2. Comalapa & 6 mujeres & $\begin{array}{l}\text { Comalapa } \\
\text { Chicomuselo }\end{array}$ & 33,3 & 0 & $\begin{array}{l}4 \text { amas de casa } \\
1 \text { modista } \\
1 \text { comerciante }\end{array}$ \\
\hline 4. Comitán & $\begin{array}{l}5 \text { mujeres } \\
1 \text { hombre }\end{array}$ & Comitán & 44,5 & $1 \mathrm{MDR}-\mathrm{TB}^{\mathrm{a}}$ & $\begin{array}{l}5 \text { amas de casa } \\
1 \text { velador }\end{array}$ \\
\hline 5. Comitán & $\begin{array}{l}2 \text { hombres } \\
3 \text { mujeres }\end{array}$ & $\begin{array}{l}\text { Comitán } \\
\text { Las Margaritas }\end{array}$ & 55,4 & 3 & $\begin{array}{l}2 \text { comerciantes } \\
1 \text { albañil } \\
1 \text { sirvienta } \\
1 \text { ama de casa }\end{array}$ \\
\hline 6. La Trinitaria & $\begin{array}{l}3 \text { hombres } \\
1 \text { mujer }\end{array}$ & La Trinitaria & 53,5 & 0 & $\begin{array}{l}2 \text { agricultores } \\
1 \text { zapatero } \\
1 \text { ama de casa }\end{array}$ \\
\hline 7. Tuxtla Gutiérrez & 7 hombres & $\begin{array}{l}\text { Tuxtla Gutiérrez } \\
\text { Chiapa de Corzo }\end{array}$ & 34,4 & 1 & $\begin{array}{l}3 \text { estudiantes } \\
1 \text { agricultor } \\
1 \text { técnico agrario } \\
1 \text { comerciante } \\
1 \text { jardinero }\end{array}$ \\
\hline 8. Tuxtla Gutiérrez & 6 mujeres & $\begin{array}{l}\text { Tuxtla Gutiérrez } \\
\text { Chiapa de Corzo }\end{array}$ & 43,5 & 2 & $\begin{array}{l}5 \text { amas de casa } \\
1 \text { sirvienta }\end{array}$ \\
\hline 11. San Cristóbal & 4 mujeres & San Cristóbal & 53,5 & 3 & $\begin{array}{l}2 \text { comerciantes } \\
2 \text { amas de casa }\end{array}$ \\
\hline Total & 62 & 10 municipios & 42,4 & 16 & \\
\hline
\end{tabular}

a MDR-TB: tuberculosis multirresistente. Las pruebas de pacientes con tuberculosis multirresistente a fármacos fueron realizadas en el Instituto Nacional de Diagnóstico y Referencia Epidemiológicas (INDRE), en la Ciudad de México.

\section{Selección de los pacientes}

Se seleccionaron los municipios de Chiapas con mayor número de casos notificados durante 1997 y 1998. Se solicitaron los listados en las unidades de salud de la Secretaría de Salud, IMSS e ISSSTE. Se visitó a cada paciente en su domicilio para solicitar su consentimiento para participar en el estudio y darle a conocer los objetivos, métodos y beneficios del mismo. Los grupos se formaron con los enfermos asistentes, que representaron $56,6 \%$ de los que aceptaron participar.

\section{Análisis de la información}

La base del análisis fueron las transcripciones completas de las entrevistas grupales y el contexto social en que viven los enfermos. Las transcripciones se leyeron en varias ocasiones para seleccionar las frases repetitivas y representativas de los pacientes, que se organizaron en una base de datos para identificar categorías y contar con campos teóricos para el análisis.

Para analizar esta investigación se eligió el modelo de búsqueda de atención propuesto por Chrisman (17), en el que se distinguen cinco etapas dife- 
rentes: 1) la definición de síntomas, que incluye la percepción que tienen los pacientes de los cambios físicos producidos por la enfermedad; 2) la conducta de los enfermos y la influencia de la evolución de los síntomas sobre la relación con sus iguales; 3) las acciones terapéuticas, que se refieren a las actividades realizadas por los pacientes para atenuar las molestias del padecimiento; 4) la consulta, la opinión de iguales acerca de los síntomas y sugerencias para su atención, y 5) la adherencia, que contempla las actividades realizadas por los pacientes para seguir el tratamiento.

Para analizar la información se diferenció entre padecimiento y enfermedad. Por padecimiento se entiende el significado que los sujetos sociales asignan a sus malestares como parte de un proceso particular que afecta a su salud, sin un diagnóstico de certeza. A su vez, la enfermedad es el conjunto de signos y síntomas físicos, psicológicos y sociales detectados a nivel clínico y que llevan al diagnóstico de un evento negativo para la salud del individuo (18). Con la enfermedad se adquieren derechos y obligaciones, y es legitimada por el grupo social y por una institución. Según las fases de este proceso de búsqueda de atención, nos referimos al padecimiento en el segundo punto de los resultados, y en los restantes a la combinación entre lo que el enfermo padece y el diagnóstico de la tuberculosis como enfermedad.

\section{RESULTADOS}

\section{Descripción general de los participantes}

En las entrevistas grupales participaron 62 pacientes (cuadro 1): 35 hombres y 27 mujeres con un promedio de 42,4 años de edad (13 a 75 años). El $41 \%$ residía en comunidades rurales y 59\% en áreas urbanas. En su mayoría, los hombres se dedicaban a la agricultura. Además de alguna actividad remunerada, todas las mujeres del medio urbano refirieron estar de- dicadas a labores del hogar, y las del medio rural colaboraban con sus esposos en la agricultura. El 90\% de los pacientes entrevistados estaban en la segunda fase del tratamiento de la tuberculosis.

\section{Identificación del padecimiento (definición de los síntomas)}

La mayoría de los pacientes comentaron que la tos fue el primer síntoma de su padecimiento, aunque inicialmente se interpretó como una gripe. Solo se pensó realmente en un padecimiento más grave después de que aparecieran hemoptisis, notable pérdida de peso, fiebre persistente y malestar general con limitación para el trabajo. $\mathrm{Al}$ inicio, los síntomas se relacionaron con el trabajo excesivo, debido al cansancio, la fiebre y el desgano que aparecía después de hacer esfuerzos físicos. Los informantes hablaron de las mojadas, polvo, humo, sol, etc., como condiciones relacionadas con los lugares de trabajo favorables a la aparición de la enfermedad. Asimismo señalaron que la mala alimentación, generalmente relacionada con la carencia de vitaminas, debilitaba el cuerpo y lo hacía propenso a la enfermedad.

\section{Diagnóstico (conducta y relación de iguales)}

La tuberculosis como enfermedad solo se identificó después del diagnóstico, es decir, después de recibir atención médica. En la mayoría de los casos esta atención no se buscó sin explorar antes otras alternativas, o hasta que la limitación para el trabajo fue muy persistente.

En un contexto rural, donde las interacciones son fundamentales, la gente refiere su vida en torno a la comunidad y el posible rechazo o distanciamiento de los conocidos tiene un impacto muy fuerte. Por lo tanto, hombres y mujeres tratan de ocultar las manifestaciones de la tuberculosis porque temen el rechazo de sus familiares y amigos por el estigma de la enfermedad.
Una de las causas del estigma de la tuberculosis fue en muchos de los casos el enfatizar demasiado los cuidados, las manifestaciones exageradas por temor al contagio y el temor a que la tuberculosis sea incurable. El temor al contagio puede llegar a ser causa de disolución o separación de la familia, siendo ello un factor negativo para el tratamiento. Los pacientes comentaron la ayuda de la información acerca de cómo se contagia la tuberculosis, su curabilidad, los cuidados necesarios para su curación, y la necesidad de que esa información se haga extensiva a todo el núcleo familiar.

El carácter contagioso de la enfermedad crea en torno al paciente un ambiente de temor por parte de los familiares y vecinos. Que digan "Ese es un tuberculoso" es un estigma para el paciente y su hogar, lo dejan de visitar, ya no le ofrecen de comer ni de beber nada, o "le buscan un traste viejo que luego pueda tirar".

La mayoría de los pacientes conocen o han escuchado historias de personas o familias completas que han muerto de tuberculosis y a quienes les han cerrado las viviendas. La gente no se acerca pensando que podría "contaminarse". Incluso en vida de esas personas enfermas, evitaban que "les regalaran algo y que los agarraran, menos".

\section{Actividades realizadas para atenuar las molestias del padecimiento}

En el estado de Chiapas es habitual el uso de tratamientos alternativos proporcionados por curanderos, pulsadores, hueseros, iloles o parteras. ${ }^{7}$ Nuestros informantes no fueron la excepción. En la mayoría de los casos recurrieron a una combinación de alternativas de atención antes de recibir tratamiento farmacológico. En algunos casos esta combinación de alternativas

\footnotetext{
$7 \quad$ El curandero puede realizar varias acciones para proteger la salud de sus pacientes, el pulsador se guía en el pulso del paciente para la realización del diagnóstico, el huesero repara huesos, el ilol protege y cura en orden de la tradición de su comunidad y la partera atiende a las mujeres durante el embarazo, parto y puerperio.
} 
incluyó los servicios de salud oficiales desde el inicio de los síntomas.

De acuerdo con los testimonios, los curanderos generalmente les recomendaron "sopladas" (el curandero toma aguardiente y lo expulsa soplando en el cuerpo del enfermo) "hierbas" o "baños" que preparan con "lociones" y hierbas, como hojas de alcanfor. Estos "baños" son de agua fría; se toman en la madrugada y durante varios días o meses. La mayoría refirieron que eso no los curó, a algunos les alivió las molestias y en otros agravó el cuadro clínico. Algunas hierbas se toman con la intención de eliminar el "mal".

Algunos curanderos recetaron medicamentos como Rifater $^{\circledR}$ (combinación de rifampicina, pirazinamida e isoniacida) o estreptomicina, que se venden libremente en las farmacias. La cantidad recetada fue una sola caja de la presentación comercial, o 3 a 10 inyecciones. Se informó del uso de otras inyecciones, de contenido desconocido, que se aplicaban en diferentes partes del cuerpo, como la espalda. Estas prácticas pueden contribuir a la aparición de tuberculosis multirresistente a los medicamentos específicos. Otras alternativas que se mencionaron fueron la ingesta de "agua de la vida" (orina de la misma persona enferma), tratamiento llamado por los informantes como orinoterapia.

\section{Atención médica}

Una vez que los enfermos de tuberculosis establecen contacto con los servicios de salud oficiales, el diagnóstico de la enfermedad puede ser más certero. Sin embargo, todos los pacientes tuvieron un retraso del diagnóstico que varió entre 3 meses y 3 años (promedio de 1 año), generalmente atribuido por ellos a un "descuido", por no pensar en la presencia de la enfermedad o por acudir en primera instancia a curanderos o hierberos: Yo lo que quería era sanar, con lo que me dieran, con lo que me den, fui con hierberos, con doctor, en los centros... ("Centros" se refiere a lugares donde atienden curanderos o brujos) (hombre, 66 años, agricultor). Por otro lado, algunos médicos tampoco pensaron que fuera tuberculosis y recetaron otros tratamientos. Además, una vez realizados los estudios específicos, hubo un atraso en la obtención de los resultados. Pero iba yo con el doctor y me decía que era asma. No pensaba que era esa enfermedad porque nunca me dio calentura, ni escalofríos, ni dolor de huesos, ni cansancio, ni rebajaba yo (mujer, 50 años, vendedora de verduras en el mercado).

Los enfermos mencionaron ventajas y desventajas del tratamiento médico. Entre las ventajas más importantes citaron la corta duración, la rápida mejoría, la gratuidad de los medicamentos y la supervisión del tratamiento que, a pesar de no ser diaria, denotaba el interés del médico. La supervisión médica se hizo cada 15 días. Ningún paciente de este estudio estuvo bajo supervisión diaria como propone la estrategia del Tratamiento Acortado Estrictamente Supervisado (TAES; DOTS en inglés), recomendada por la Organización Mundial de la Salud (OMS) y que consiste en que un supervisor observe al paciente mientras ingiere los comprimidos (19).

Entre las desventajas que condujeron a la falta de adherencia al tratamiento, mencionaron que eran demasiados antibióticos y que les produjeron efectos colaterales, como gastritis, sudación y mareos. Otra causa fue que los pacientes tuvieron gastos de pasaje y alimentación para recoger su medicación para cada semana, quincena o mes. Hubo una paciente que ocultó su enfermedad al esposo y terminó el tratamiento con muchos problemas de pareja, ya que este le prohibió asistir al centro de salud por celos.

Aunque todos los enfermos estaban recibiendo tratamiento farmacológico, refirieron tener otros problemas con el servicio de salud, relacionados sobre todo con el retraso del diagnóstico y la falta de medicamentos. Algunos pacientes que ya tenían conocimiento de problemas en la atención de los servicios públicos de salud prefirieron acudir en primera instancia a médicos particulares. Los comentarios de otros enfermos sobre la mala calidad de la atención, aunados a lo avanzado de la enfermedad, representaron una dificultad mayor al decidir a qué servicios médicos acudir. La visita oportuna a los servicios de salud públicos se vio afectada por la idea de tener que perder un día de trabajo, ya que en los hospitales y unidades de salud urbanas los enfermos deben llegar muy temprano, obtener fichas y esperar mucho tiempo, por el número limitado de consultas.

Existe también una serie de medidas generales que pueden ser indicaciones del médico, recomendaciones de familiares y vecinos o derivadas del conocimiento de los mismos pacientes sobre las enfermedades respiratorias. Entre ellas destacan: comer bien, no tomar aguardiente, no comer picante, separar los trastos del enfermo, no tomar agua fría, no bañarse con agua fría, no comer carne de puerco, no salir al frío, no enojarse, no tener relaciones sexuales y "dieta". Para los pacientes del medio rural, "dieta" es un concepto amplio que incluye algunas o todas las recomendaciones mencionadas $\mathrm{y}$, principalmente, la abstinencia sexual durante el tratamiento, que en caso de incumplimiento se consideró como causa de recaídas o fracasos del tratamiento.

\section{El tratamiento y la adherencia al mismo}

El principal problema referido por los pacientes para no asistir al servicio de salud o no cumplir el tratamiento médico fue la necesidad de continuar trabajando debido a sus necesidades económicas de sustento familiar.

Los campesinos propietarios de tierras tuvieron más posibilidades de reanudar su trabajo. En el caso de la producción artesanal, bastante general en los Altos de Chiapas, la familia se hizo cargo de la producción durante la enfermedad de alguno de sus miembros. En este contexto, cualquier miembro de la familia tiene más posibilidades de sobrevivir, por la solidaridad que la misma forma de producir genera. El problema se presenta cuando una sola 
persona es la responsable del ingreso económico y no tiene la propiedad de los medios de producción, como fue el caso de los asalariados, vendedores ambulantes, albañiles, mujeres solteras con hijos, amas de casa, chóferes y jornaleros agrícolas, que fueron quienes encontraron mayores dificultades para recibir el tratamiento antituberculoso. La migración por motivo de trabajo o de conflictos sociopolíticos también fue desfavorable para el cumplimiento del tratamiento, ya que no siempre se comunica a la institución de salud para trasladar el tratamiento a donde sea factible. En unidades productivas familiares, la propia familia asume la responsabilidad del paciente. El sentirse útil, necesario y querido es un motivo para tomar el tratamiento, curarse y, consecuentemente, trabajar.

La historia individual y social de los pacientes chiapanecos es una historia de dificultades, obstáculos y pobreza. La vida para ellos es un escenario donde solo el trabajo les puede ayudar a sobrevivir. Debido a la tuberculosis, algunos dejaron de trabajar o trabajaron con grandes esfuerzos. Ir al centro de salud significaba no poder darle continuidad a su trabajo. La falta de ingresos necesarios para subsistir produjo en la mayoría una mayor depresión, aparte del estado físico en que ya se encontraban.

Los pacientes que refirieron haber abandonado el tratamiento mencionaron que, al acudir a médicos particulares y debido a escasez de recursos para comprar sus medicamentos, dejaron de tomarlos. Otras causas fueron los efectos colaterales, como la gastritis, los mareos y las molestias de las inyecciones intramusculares, como en el caso de la estreptomicina. Otra de las causas detectadas fue la falta de comunicación entre el médico y el paciente. Hubo incluso algunos pacientes que no reconocieron haber abandonado el tratamiento, alegando que sólo siguieron las instrucciones del médico.

\section{DISCUSIÓN}

Los problemas para el diagnóstico temprano y el tratamiento oportuno de la tuberculosis en Chiapas están relacionados con las percepciones que los enfermos tienen sobre los cambios que la enfermedad produce en su vida cotidiana y las alternativas de atención que eligen, de acuerdo con sus creencias y posibilidades económicas.

El proceso de atención y curación de la enfermedad es habitualmente prolongado, ya que generalmente el diagnóstico sufre retraso y se abandona el tratamiento. En la clase social de los pacientes, puede advertirse que las condiciones del trabajo propician la enfermedad y que, en la medida en que persistan los bajos salarios y el desempleo, habrá menos posibilidades de éxito de los tratamientos y del control de la tuberculosis.

Después de un ir y venir por diferentes alternativas de alivio, los pacientes llegan a los servicios médicos, en los que la estrategia actual es el TAES. Sin embargo, ninguno de nuestros informantes recibió tratamiento supervisado diariamente. Las causas son obvias y están relacionadas con las dificultades mencionadas en este estudio. En algunos casos, cuando la relación de los pacientes con el médico o la enfermera se establece adecuadamente, se utilizan otras alternativas de supervisión contempladas en el TAES, como la participación de la familia, que resultó en el éxito del tratamiento.

La detección y diagnóstico de los casos de tuberculosis plantea serios problemas que persisten durante el tratamiento, con la consiguiente falta de adherencia al esquema recomendado. No es posible predecir el abandono en función de factores específicos relacionados con los servicios de salud o variables demográficas, socioeconómicas y culturales de los individuos, ya que estos factores se combinan de numerosas formas, tantas como estilos de vida de cada individuo. En este sentido, la tarea de los servicios de salud debería encaminarse a incrementar la calidad con conocimiento del contexto social y cultural del estado, a establecer una mejor relación médico-paciente $(20,21)$, a proceder de forma sistemática al estudio de los pacientes que consultan con tos y a ofrecer alternativas de supervi- sión del tratamiento en la localidad de residencia del enfermo.

Existen otras estrategias para incrementar la adherencia al tratamiento de la tuberculosis, tales como incentivos financieros, motivación, educación y hospitalización, pero son más eficaces la supervisión diaria del tratamiento y la combinación de dos o más de estas estrategias. Esto ya se ha evaluado en países donde la falta de adherencia está relacionada principalmente con el consumo de drogas, la infección por el VIH y la indigencia (22-25). Muy diferentes son las condiciones del país y, más aún, el panorama de la población rural enferma de tuberculosis en Chiapas. Durante 1996, con el apoyo de las autoridades nacionales, estatales y locales, se aplicó la estrategia en Chiapas en pequeñas áreas de las jurisdicciones sanitarias, alcanzando una eficacia de $98 \%$ y una eficiencia de $88 \%$ (26). Sin embargo, no pudo ampliarse a la totalidad del estado e incluso en esas áreas ya no se aplica con las características y criterios recomendados por la OMS; según los datos del primer semestre de 1998, la eficiencia en las áreas consideradas de aplicación del TAES, pero en las que en realidad ya no se aplica, fue de $57,2 \%$, frente a $69,1 \%$ en las áreas de tratamiento autoadministrado. ${ }^{8}$

Es conveniente utilizar un enfoque multidisciplinario en el control de la enfermedad que incluya: 1) la participación de la comunidad, involucrando a personajes comunitarios en la elaboración de los programas de prevención y control, y especialmente en la supervisión del tratamiento; 2) un programa de capacitación permanente del personal médico y paramédico, además de mejorar la relación del médico con el enfermo, y 3) facilitar el conocimiento de la enfermedad, mediante difusión amplia en los medios de comunicación, como la televisión y la radio.

La tuberculosis es un problema social, pero puede controlarse a pe-

\footnotetext{
8 Instituto de Salud del Estado de Chiapas. Programa de Prevención y Control de la Tuberculosis en Chiapas, 2000
} 
sar de las condiciones socioeconómicas de los enfermos (27). Es necesario procurar una mejor calidad y acceso a los servicios de salud. De otra manera, la transmisión continuará y el riesgo será mayor debido a la aparición de cepas resistentes que no pue-

1. Schluger NW. The impact of drug resistance on the global tuberculosis epidemic. Int J Tuber Lung Dis 2000;4:71-75.

2. Houston S, Fanning A. Current and potential treatment of tuberculosis. Drugs 1994;48: 689-708.

3. Culliton BJ. Drug-resistant TB may bring epidemic. Nature 1992;356:473.

4. Small PM, Shafer RW, Hopewell PC, Singh SP, Murphy MJ. Exogenous reinfection with multidrug-resistant $M$. tuberculosis in patients with advanced HIV infection. N Eng J Med 1993;328:1137-1144.

5. Rouillon A, Pedrizet S, Parrot R, Waaler H. La transmisión del bacilo tuberculoso: el efecto de la quimioterapia. En: Publicación Científica No. 346, OPS, OSP 1977;1-28.

6. Cano-Pérez G. Evaluación de los esquemas de tratamiento primario de tuberculosis en servicios de la Secretaría de Salud. Neumología y Cirugía de Tórax 1987;XVLI:6-11.

7. México, Secretaría de Salud. Manual de procedimientos del Programa Nacional de Prevención y Control de la Tuberculosis. México. D.F.: Secretaría de Salud de México; 1999.

8. México, Instituto de Salud del Estado de Chiapas. Programa de Prevención y Control de la Tuberculosis en Chiapas. Tuxtla Gutiérrez, Chiapas: Instituto de Salud del Estado de Chiapas; 1997.

9. México, Secretaría de Hacienda, Gobierno del Estado de Chiapas. Agenda Estadística de Chiapas. Tuxtla Gutiérrez, Chiapas: Secretaría de Hacienda, Gobierno del Estado de Chiapas; 1997.

10. México, Instituto de Salud del Estado de Chiapas, Dirección de Planeación, Información y Evaluación. Catálogo de Unidades Médicas. dan ser tratadas con los medicamentos existentes.

Agradecimientos. Este proyecto de investigación fue financiado por el Sistema de Investigación Benito Juárez (SIBEJ) de Oaxaca, Oax, México. Se

\section{REFERENCIAS}

Tuxtla Gutiérrez, Chiapas: Instituto de Salud del Estado de Chiapas; 1999.

11. Menegoni L. Conceptions of tuberculosis and therapeutic choices in Highland Chiapas, México. Med Anthropol Q 1996;10:381-401.

12. Fabrega $H$, Silver DB. Illness and shamanistic curing in Zinacantan. An ethnomedical analysis. Stanford, CA: Stanford Univrsity Press; 1973. pp. 81-126.

13. Solórzano JJ. Seguimiento de tuberculosis: estudio de 106 casos a tres años de su alta. Presentado en el II Congreso Nacional de Salud Pública, Morelos, México. 1991.

14. Menéndez LE. Estilos de vida, riesgos y construcción social. Concepto similares y significados diferentes. Estudios Sociológicos 1998; XVI:37-67.

15. Rojas Soriano R. Crisis, salud-enfermedad y práctica médica. $1^{a}$ ed. México, D.F.: Plaza y Valdés; 1990. pp. 22-27.

16. Rojas Soriano R. Sociología Médica. México, D.F.: Plaza y Valdés; 1988. pp. 7-57.

17. Chrisman N. The health-seeking process: an approach to the natural history of illness. Culture, Medicine and Psychiatry 1977;1:351-377.

18. Módena ME. Cultura, enfermedad-padecimiento y atención alternativa. La construcción social de la desalcoholización. En: Salud, cambio social y política. México, D.F.: EDAMEX; 1999. pp. 383-400.

19. Organización Mundial de la Salud. Tratamiento de la tuberculosis. Directrices para los Programas Nacionales. Programa Mundial contra la Tuberculosis. Ginebra: OMS; 1997.

20. Jaramillo E. Pulmonary tuberculosis and health-seeking behaviour: how to get a delayed diagnosis in Cali, Colombia. Trop Med Int Health 1998;3:138-144. agradece el apoyo de la Secretaría de Salud, del Instituto Mexicano del Seguro Social y del Instituto de Servicios y Seguridad Social para los Trabajadores del Estado, por todas las facilidades prestadas al buen desarrollo de esta investigación.
21. Jaramillo E. Tuberculosis control in less developed countries: can culture explain the whole picture? Trop Doct 1998;28:196-200.

22. Volmik J, Garner P. Systematic review of randomised controlled trials of strategies to promote adherence to tuberculosis treatment. $\mathrm{Br}$ Med J 1997;315:1403-1410.

23. Singleton L, Turner M, Haskal R, Etkind S, Tricarico M, Nardell E. Long-term hospitalization for tuberculosis control. Experience with a medical psychosocial inpatient unit. JAMA 1997;278:838-842.

24. Marco A, Caylá JA, Serra M, Pedro R, Sanrama $C$, Guerrero $R$, Ribot N. Predictors of adherence to tuberculosis treatment in a supervised therapy programme for prisoners before and after release. Study Group of Adherence to Tuberculosis Treatment of Prisoners. Eur Respir J 1998;12:967-971.

25. Cohen FL. Adherence to therapy in tuberculosis. Annu Rev Nurs Resp 1997;15:153-184.

26. Álvarez-Gordillo GC, Dorantes-Jiménez JE. Tratamiento acortado estrictamente supervisado para tuberculosis pulmonar en Chiapas. Salud Publica Mex 1998;40:272-275.

27. Organización Panamericana de la Salud/ Organización Mundial de la Salud. Perú. Programa de control de la tuberculosis. ¿Por qué un ejemplo para el mundo?. Washington, D.C.: OPS/OMS; 1997.

Manuscrito recibido el 21 de noviembre de 2000. Aceptado para publicación, tras revisión, el 25 de abril de 2001. 


\title{
ANEXO 1. Guía de la entrevista
}

\author{
Concepto de salud-enfermedad \\ 1. ¿Cómo se sienten en este momento? \\ 2. ¿Cómo es eso de sentirse bien? \\ 3. ¿Cómo es eso de sentirse mal? \\ 4. ¿Cuándo se dice que alguien está enfermo? \\ 5. ¿Por qué creen que las personas se enferman?
}

Diagnóstico de la tuberculosis

a. ¿Qué han oído acerca de la tuberculosis?

b. ¿Por qué será que las personas se enferman de tuberculosis?

c. ¿Cómo supieron ustedes que tenían tuberculosis?

d. ¿Hay otras personas en su casa con la misma enfermedad?

e. ¿Conocen a otras personas en su comunidad con tuberculosis?

f. ¿Cómo les empezó la enfermedad?

g. ¿Qué pensaron cuando supieron que tenían tuberculosis?

h. ¿Cuáles fueron sus sentimientos al comprender que tenía tuberculosis? ¿Qué clase de emociones tuvieron? ¿tuvieron depresión?

i. ¿A quien acudieron la primera vez que se sintieron mal?

j. ¿Tuvieron alguna dificultad para recibir atención médica?

k. ¿Cómo les dijo el médico que tenían tuberculosis?

Tratamiento de la tuberculosis

1. ¿Qué cuidados han tenido durante la enfermedad?

2. ¿A que lugares han acudido para que los atiendan?

3. ¿Cuáles son los tratamientos que conocen?

4. ¿Creen que la tuberculosis es curable?

5. ¿Qué medicinas han tomado?

Abandono del tratamiento

1. ¿Alguien ha recibido otros tratamientos de medicinas antes del que actualmente toman?

2. ¿Alguno de ustedes ha dejado de tomar las medicinas?

3. ¿Conocen a algunas personas que dejan de tomar sus medicinas?

4. ¿Qué piensan de ellos?

5. ¿Han tenido molestias al tomar la medicina?

6. ¿Han tenido algún problema para conseguir o recoger la medicina en el Centro de Salud?

7. ¿Alguien lo ha tratado mal y hasta ha pensado no regresar?

Relaciones con la pareja y la familia

1. ¿Cómo ha sido la vida con sus hijos a partir de su enfermedad?

2. ¿Cómo ha sido la vida con su pareja?

3. ¿Qué han hecho sus familiares desde que está enfermo?

4. ¿Cómo lo tratan sus vecinos en su comunidad?

5. ¿Ha sentido algún rechazo de las personas que saben que está enfermo?

6. ¿Cómo afectó la tuberculosis a su estilo de vida?

Relación médico-paciente

1. ¿Entiende lo que le dice el médico acerca de la tuberculosis?

2. ¿Le tiene confianza al médico?

3. ¿Le pregunta todas las dudas que tiene?

4. ¿Alguna vez ha tenido problemas con algún médico?

5. ¿Le ha molestado algo que le diga o haga el médico?

6. ¿Le ha dicho el médico, los cuidados que debe tener durante el tratamiento? 
ABSTRACT Objective. To analyze the process of seeking tuberculosis care and this process's impact on treatment adherence in the Mexican state of Chiapas, given that the two primary factors in successful tuberculosis (TB) control programs are early diagnosis and adherence to TB treatment.

\title{
Seeking tuberculosis care in Chiapas, Mexico
}

Methods. We conducted a qualitative study using group interviews with 11 groups of patients in three of the nine socioeconomic regions of the state of the Chiapas (Altos, Centro, and Fronteriza).

Results. The patients applied a variety of approaches in seeking care. The patients reported considerable delays in diagnosis due to problems that the patients themselves had and because of shortcomings in the care they received from the formal health care system. The treatment options that they followed were the result of their perceptions of the causes of TB and of the variety of traditional medical practices accepted in their communities.

Conclusions. The lack of knowledge about TB encourages people to consider various alternatives for their care. Tuberculosis control in Chiapas requires an optimal utilization of the health services that exist in the state as well as a program of health education. TB control in Chiapas must take into account the social, cultural, and economic reality of the population.

\section{Jornadas Australes Interdisciplinarias Mujer y Desarrollo: Construyendo el Derecho a la Igualdad}

\author{
Fechas: $\quad 4$ a 8 de diciembre de 2001 \\ Lugar: $\quad$ Valdivia, Chile
}

La Universidad Austral de Chile se complace en anunciar la celebración de estas jornadas, destinadas a proporcionar un ámbito propicio para la reflexión, discusión y análisis de los adelantos obtenidos en la incorporación de los asuntos relacionados con el género en las políticas públicas, la investigación y la formación de recursos. En las jornadas también se plantearán las tareas específicas que deben llevarse a cabo para que la mujer pueda participar plenamente en la vida política y social, como sujeto con igualdad de derechos, y para que las relaciones entre los géneros se produzcan con equidad, justicia y armonía.

Habrá discusiones en grupo, conferencias y cursos sobre los siguientes temas: 1) salud reproductiva; 2) metodología de la investigación de tipo cualitativo para estudiar la salud sexual y reproductiva; 3 ) avances conceptuales y metodológicos en la construcción, sistematización y comparación de indicadores de género; 4) promoción y monitoreo de los derechos sexuales y reproductivos; 5) monitoreo del Programa de Cáncer Cervicouterino; 6) la salud mental de la mujer; 7) la mujer y el climaterio; 8) los derechos desde el punto de vista del género.

La fecha límita para la recepción de trabajos es el 31 de julio de 2001.

\section{Información:}

Comité II Jornadas Australes Interdisciplinarias Mujer y Desarrollo

Instituto de Enfermería Materna, Facultad de Medicina

Universidad Austral de Chile

Casilla 567

Valdivia, Chile

Correo electrónico: Jmujer@uach.cl 